\title{
Phonon resonances in photoluminescence spectra of self-assembled quantum dots in an electric field
}

\author{
Ivan V. Ignatiev* and Igor E. Kozin \\ Single Quantum Dot Project, ERATO, JST, Tsukuba Research Consortium, Tsukuba 300-2635, Japan \\ and Institute of Physics, St.-Petersburg State University, St.-Petersburg, Russia \\ Valentin G. Davydov, ${ }^{\dagger}$ Selvakumar V. Nair, Jeong-Sik Lee, and Hong-Wen Ren ${ }^{\ddagger}$ \\ Single Quantum Dot Project, ERATO, JST, Tsukuba Research Consortium, Tsukuba 300-2635, Japan \\ Shigeo Sugou \\ Opto-electronics Research Laboratories, NEC Corporation, Tsukuba 305-0841, Japan \\ and Single Quantum Dot Project, ERATO, JST, Tsukuba Research Consortium, Tsukuba 300-2635, Japan \\ Yasuaki Masumoto \\ Single Quantum Dot Project, ERATO, JST, Tsukuba Research Consortium, Tsukuba 300-2635, Japan \\ and Institute of Physics, University of Tsukuba, Tsukuba 305-8571, Japan
}

(Received 20 May 2000; revised manuscript received 11 September 2000; published 31 January 2001)

\begin{abstract}
Phonon resonances observed in the photoluminescence (PL) spectra of InP and $\operatorname{In}_{0.35} \mathrm{Ga}_{0.65} \mathrm{As}$ selfassembled quantum dots (QD's) in an external electric field are studied in detail. The resonances are shown to arise from fast phonon-assisted relaxation of hot carriers, and to become observable when the PL is quenched by nonradiative losses from excited states. A simple model is developed that considers tunneling of the carriers from the QD's into the barrier layer as the main process responsible for PL quenching in the presence of an electric field. From this model, the depth of the potential well for holes is estimated to be $10-20 \mathrm{meV}$ for the InP QD's. The PL kinetics measurement is performed with a time resolution of 6 ps. Clear evidence of surprisingly fast carrier relaxation with emission of high-energy acoustic phonons is found. Further acceleration of the carrier relaxation is observed under strong optical pumping. We consider this effect to be caused by Auger-like carrier-carrier scattering processes. Acceleration of the relaxation observed at elevated temperatures is ascribed to stimulated phonon emission.
\end{abstract}

DOI: 10.1103/PhysRevB.63.075316

PACS number(s): 78.66.Fd, 63.22. $+\mathrm{m}, 78.47 .+\mathrm{p}, 73.21 .-\mathrm{b}$

\section{INTRODUCTION}

Quantum dots (QD's) attract much attention because of their unusual physical properties and promising prospects for application in device engineering. Three-dimensional carrier confinement gives rise to discrete energy levels with large spacings, provided that the QD's are sufficiently small. For this reason, QD's are often referred to as artificial atoms. ${ }^{1}$ Realization of such artificial atoms in semiconductor heterostructures and their integration with conventional electronic and optical devices offer great potential for applications. ${ }^{2-4}$ For optical applications, the main physical processes are the creation of carriers in the QD, their relaxation to the lowestenergy states, and radiative recombination.

The carrier relaxation process was studied extensively during the last decade because it mainly determines the luminescence efficiency of QD's. ${ }^{5-12}$ At low temperature and weak excitation, the main relaxation mechanism is spontaneous emission of phonons by hot carriers. Though this point is evident, peculiarities of this process in QD's are not yet understood and have been widely discussed up to now. ${ }^{13-23}$

In polar semiconductors of interest, electrons and holes interact strongly only with the longitudinal-optical (LO) phonons. LO phonons produce an electric field to which the electrons and holes are sensitive as charged particles. The energy spectrum of the LO phonons is typically rather narrow, and can be roughly considered as a set of degenerate energy values. Therefore, fast relaxation occurs only in those QD's where the energy spacings are equal to the energy of one or several LO phonons. The carriers in other QD's have to relax via acoustic-phonon emission. This process is inherently slow because of the weak deformation potential interaction. This is well known for bulk materials, where the relaxation with emission of acoustic phonons is slower by two orders of magnitude than that with emission of LO phonons. $^{24}$ Theoretical considerations show that acousticphonon-mediated relaxation is further slowed down in QD's-this effect is commonly referred to as "phonon bottleneck." ${ }^{, 5,6}$ Only phonons with wavelengths of the order of the QD diameter and an energy equal to an interlevel spacing may cause one-phonon relaxation. Although two-phonon relaxation is not so restricted, this process should be also slow because of weak electron-phonon interaction. $^{7}$

It seems that some indication of the phonon bottleneck is found in a few experiments. The simplest experiment consists of the observation of the photoluminescence (PL) spectrum of a QD ensemble under an optical excitation slightly above the PL maximum. Sharp features (resonances) shifted by the LO-phonon energy from the excitation line were observed in the spectra of some samples. ${ }^{13,14,16,18,20,25-27}$ 
Other types of experiments apparently argue against the phonon bottleneck. The most important of these is the study of the rise time of the PL, that is determined by the relaxation of hot carriers to their lowest energy levels. These experiments show a fairly short rise time of a few or several tens of picoseconds in high-quality samples. ${ }^{17,18}$ This observation contradicts theoretical predictions. However, in such experiments it is difficult to achieve both good spectral and time resolutions keeping a low excitation power. Therefore, it is not clear whether the observed fast relaxation is caused by LO-phonon emission, by acoustic-phonon emission, or by some other processes.

Many theoretical efforts have been put forward to explain fast carrier relaxation. Two-phonon processes involving LO \pm LA combination, ${ }^{7}$ LO-phonon anharmonicity ${ }^{21}$ relaxation through continuum states, ${ }^{22}$ and defect states ${ }^{12,28,29}$ were considered to spread out the spectral region where the fast phonon-assisted relaxation is possible. In addition, an alternative mechanism of relaxation was proposed that considered carrier-carrier scattering (referred to as Auger-like processes). ${ }^{8,11,30-32}$

We recently proposed a powerful method to study carrier relaxation in QD's. ${ }^{23}$ This method exploits the idea of artificially controlling PL quenching. ${ }^{33-35}$ The PL quenching rate can be changed in a wide range by applying an external electric field to the sample. This allows one to study carrier relaxation by varying the ratio of the relaxation and PL quenching rates.

In this paper, we use this method to study phonon-assisted carrier relaxation in self-assembled QD's in more detail. We study the PL spectra and PL kinetics of heterostructures with InP and $\mathrm{In}_{0.35} \mathrm{Ga}_{0.65}$ As QD's in an electric field under quasiresonant excitation. We find that, for all heterostructures studied, a fast LO-phonon-assisted relaxation gives rise to LO resonances in the spectra of PL partially quenched by an electric field. The acoustic-phonon-assisted relaxation is not suppressed as strongly as predicted theoretically. The interaction with high-energy acoustic phonons is also fairly strong, and gives rise to a relatively fast relaxation which is only an order of magnitude slower than the resonant LOphonon-assisted relaxation. We discuss a possible physical mechanism of the relatively strong interaction of an electronhole pair with high-energy acoustic phonons. According to our results, we conclude that there is no phonon bottleneck effect in QD's, at least in the sense that it was proposed theoretically.

We present a model for a PL quenching mechanism in the presence of an electric field. The essential point of our model is the tunneling of carriers from QD's into the barrier layer. From a quantitative analysis of the experimental data, we evaluated the depth of the potential well for holes in InP QD's.

We also discuss an acceleration of the carrier relaxation due to carrier-carrier scattering and stimulated phonon emission. For this purpose, we study the PL spectra of samples in an electric field under strong optical pumping and at elevated temperatures.

\section{EXPERIMENTAL DETAILS}

The sample with InP QD's was grown by gas-source molecular-beam epitaxy on an $n^{+}$GaAs substrate. A 300-nm GaAs buffer layer containing a thin 3 $\times(2-n m$ AlAs/10-nm GaAs) superlattice in the middle was grown at $600^{\circ} \mathrm{C}$ to suppress dislocations. A thin $(2 \mathrm{~nm})$ AlAs layer on the buffer layer was grown to prevent compositional interdiffusion between $\mathrm{GaAs}$ and $\mathrm{In}_{0.5} \mathrm{Ga}_{0.5} \mathrm{P}$ layers. One layer of InP QD's with a nominal thickness of $4 \mathrm{ML}$ was grown between the $100-\mathrm{nm} \mathrm{In}_{0.5} \mathrm{Ga}_{0.5} \mathrm{P}$ barrier layers. The growth rates were $0.5 \mathrm{ML} / \mathrm{s}$ for $\mathrm{In}_{0.5} \mathrm{Ga}_{0.5} \mathrm{P}$, and 0.25 $\mathrm{ML} / \mathrm{s}$ for InP. The interruption times used before and after the InP growth were 2 and $20 \mathrm{~s}$, respectively. The areal density of the QD's is about $10^{10} \mathrm{~cm}^{-2}$. This was determined by atomic force microscopy on a reference sample, grown in the same conditions without the top barrier layer. The average base diameter of the QD's is $\approx 40 \mathrm{~nm}$ and the height is $\approx 5 \mathrm{~nm}$, as determined by cross-sectional transmission electron microscopy. The sample with $\operatorname{In}_{0.35} \mathrm{Ga}_{0.65}$ As QD's was grown by metal organic vapor phase epitaxy on the (711)B $n^{+}$substrate. A QD layer with a nominal thickness of 4.5 MLs is embedded between 250- and 100-nm GaAs layers containing 2-nm AlAs layers as stop layers for photocreated carriers. The areal density of the QD's is about 5 $\times 10^{9} \mathrm{~cm}^{-2}$. The average base diameter is $\approx 70 \mathrm{~nm}$, and the height is $\approx 10 \mathrm{~nm}$ as determined by atomic force microscopy of the reference sample. The samples were provided with a semitransparent gold or indium tin oxide Shottky contact on the top surface, and with an Ohmic contact on the back surface.

The PL was excited selectively by a continuous wave (cw) Ti:sapphire laser within or slightly above the PL band of the QD's. This excitation is hereafter referred to as quasiresonant excitation. The pump power density was less than $100 \mathrm{~W} / \mathrm{cm}^{2}$. We checked that the PL intensity depended linearly on the excitation power within this range without any change of the PL spectrum. The spectral width of the laser line was less than $0.01 \mathrm{meV}$ without any sidebands. The PL was dispersed by a double monochromator U1000 (focal length $1 \mathrm{~m}$, numerical aperture $1: 8$, linear dispersion 0.36 $\mathrm{nm} / \mathrm{mm}$ ) with an extremely low background of scattered light $\left(10^{-14}\right.$ at a distance of $3 \mathrm{meV}$ from the laser line). The spectral resolution of the setup was approximately $0.15 \mathrm{meV}$. A photon counting system with a cooled GaAs or $\mathrm{In}_{0.35} \mathrm{Ga}_{0.65} \mathrm{As}$ photomultiplier tube was used for detection of the PL signal.

The PL kinetics was studied using a picosecond Ti:sapphire laser with a pulse duration ranging from 1 to $5 \mathrm{ps}$, and a repetition rate of $82 \mathrm{MHz}$. In most experiments, we used a fairly low average pump power density of about $50 \mathrm{~W} / \mathrm{cm}^{2}$ to prevent the creation of more than one electron-hole pair in a QD per pulse. This allowed us to avoid relaxation due to carrier-carrier scattering. A $0.25-\mathrm{m}$ double-subtractive dispersion monochromator (spectral resolution $0.5 \mathrm{~nm}$ ) and a streak camera were used for accumulation of the signal in the selected spectral points. The time resolution of the setup was 


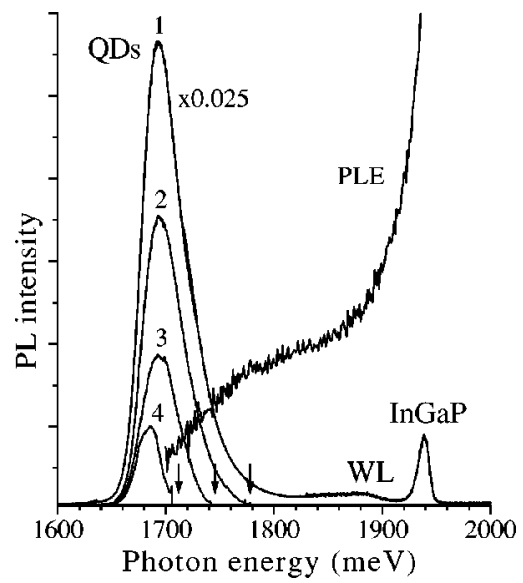

FIG. 1. The PL and photoluminescence excitation (PLE) spectra of the sample with InP QD's. PL spectrum 1 was recorded under $\mathrm{In}_{0.5} \mathrm{Ga}_{0.5} \mathrm{P}$ band-to-band excitation. It is scaled by a factor 0.025 . The PL bands of the $\mathrm{In}_{0.5} \mathrm{Ga}_{0.5} \mathrm{P}$ barriers, the wetting layer, and the InP QD's are marked. PL spectra 2, 3, and 4 were recorded under quasiresonant excitation with photon energies indicated by arrows. The PLE spectrum was recorded by $\mathrm{PL}$ detection at $E_{\mathrm{PL}}$ $=1700 \mathrm{meV}$.

about 6 ps. Most measurements were done at a sample temperature $2 \mathrm{~K}$ to exclude any processes involving absorption or stimulated emission of phonons.

\section{EXPERIMENTAL RESULTS AND DISCUSSION}

\section{A. PL spectra}

The PL bands of the heterostructures recorded under nonresonant (barrier band-to-band) and quasiresonant excitation have a smooth profile without any sharp features. A typical spectrum of the sample with InP QD's is shown in Fig. 1. As seen, the most intense PL band in the spectrum is that of the QD's. The PL profile does not contain sharp features for any photon energy of excitation. The PLE spectrum is also smooth.

When a negative bias is applied to the sample surface, the integral PL intensity decreases. At the same time, the decrease of the PL in different spectral points is different and, as a result, pronounced resonances appear in the PL spectra. This behavior of the spectra was already discussed in Ref. 23. An example of the PL spectra of the InP QD's for various excitation energies is shown in Fig. 2. As seen, many resonances appeared in the spectra at negative bias. The most prominent resonance is shifted approximately from the excitation line by the LO phonon energy of the bulk InP crystal $\left[\hbar \omega_{\mathrm{LO}}=43.5 \mathrm{meV}\right.$ (Ref. 36)]. The energy positions of the resonances faithfully follow the photon energy of the excitation. Under the excitation at a high photon energy, a resonance shifted by $2 \hbar \omega_{\mathrm{LO}}$ (marked by $2 \mathrm{LO}$ in Fig. 2 ) is also observed. The relative intensity of the $1 \mathrm{LO}$ and $2 \mathrm{LO}$ resonances depends strongly on the excitation energy. The intensity of the resonances becomes weak when they leave a PL band measured at zero bias.

A few other weak resonances are visible in the spectral region between the excitation line and the $1 \mathrm{LO}$ resonance

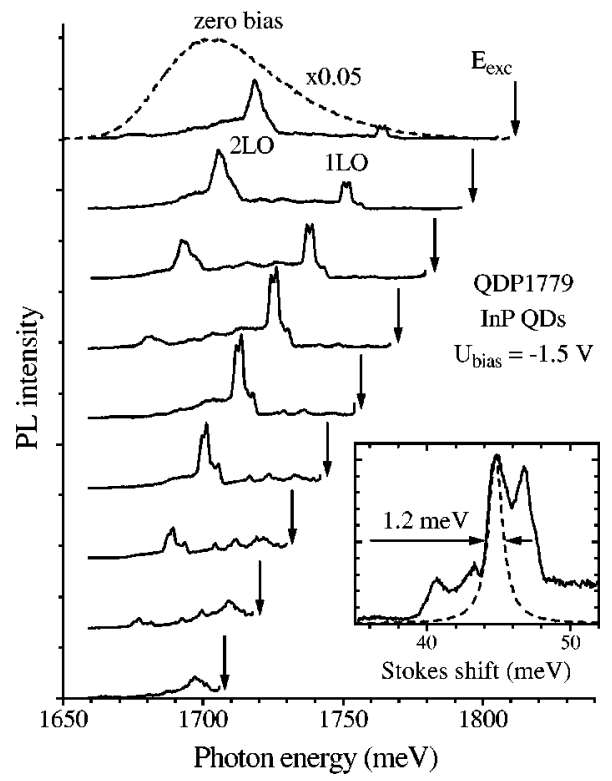

FIG. 2. PL spectra of the sample with InP QD's at different excitations indicated by arrows. $U_{\text {bias }}=-1.5 \mathrm{~V}$. The spectra are shifted vertically for clarity. The top curve, shown by a dashed line, is the spectrum at zero bias. It is scaled by a factor of 0.05 . Inset: a part of the PL spectrum showing an LO resonance vs the Stokes shift. A fit of one of the peaks in the resonance by a Lorentzian is shown by dashed lines.

(hereafter referred to as the acoustic region of the spectrum), and also between the 1LO and 2LO resonances. Their energy shifts from the excitation line or the $1 \mathrm{LO}$ resonance line coincide with the energies of high-energy transverse acoustic (TA) and longitudinal acoustic (LA) phonons in InP crystal.

PL spectra of the sample with $\operatorname{In}_{0.35} \mathrm{Ga}_{0.65}$ As QD's are shown in Fig. 3. Without bias, the PL spectrum consists of two smooth maxima (see the inset), probably due to the existence of two sets of QD's with different sizes in this sample. A number of maxima (resonances) appear in the spectrum at negative bias. The most prominent resonances can be assigned to the GaAs- and InAs-like LO phonons of the $\mathrm{In}_{0.35} \mathrm{Ga}_{0.65} \mathrm{As}$ QD's, because their energy shifts from the excitation line are close to the energy of the LO phonons in GaAs and InAs crystals, respectively. They are marked in Fig. 3 as $\mathrm{LO}_{G}$ and $\mathrm{LO}_{I}$. An intense resonance with a Stokes shift of about $20 \mathrm{meV}$ can be assigned to LA phonons of an $\mathrm{In}_{0.35} \mathrm{Ga}_{0.65} \mathrm{As}$ solid solution. A narrow peak with a Stokes shift of $33.2 \mathrm{meV}$, marked by * in Fig. 3, is probably due to the Raman scattering from the GaAs barrier layers, because the intensity of this peak does not depend on bias.

The LO-phonon resonances for all the samples have a rather complicated structure consisting of a few narrow peaks. A fit of each separate peak in the resonance by Lorentzians allows one to estimate the full width at half maximum (FWHM) to be about $1.2 \mathrm{meV}$ for the InP QD's, as shown in the inset of Fig. 2. A similar fit of the $\mathrm{LO}_{G}$ resonance in the spectra of the $\mathrm{In}_{0.35} \mathrm{Ga}_{0.65}$ As QD's (see Fig. 3) gives a FWHM of $0.5 \mathrm{meV}$.

Under positive bias, the PL intensity also starts to decrease. However, for $U_{\text {bias }}>0.7 \mathrm{~V}$, a strong increase of the 


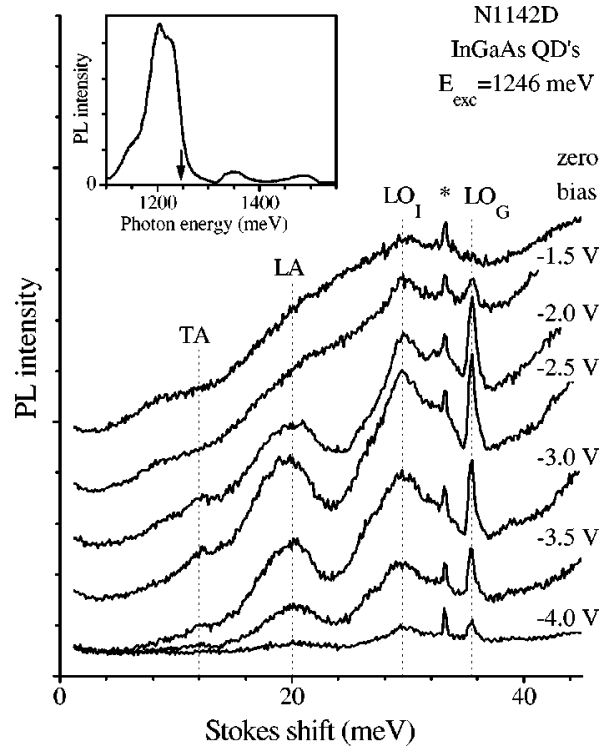

FIG. 3. The PL intensity vs the Stokes shift for the sample with $\mathrm{In}_{0.35} \mathrm{Ga}_{0.65} \mathrm{As}$ QD's under different biases indicated against each curve. $E_{\mathrm{exc}}=1246 \mathrm{meV}$. The spectra are shifted vertically for clarity. The energies of the resonances marked by TA, LA, $\mathrm{LO}_{I}$, and $\mathrm{LO}_{G}$ are $12,20,30$, and $35.5 \mathrm{meV}$, respectively. Inset: PL spectrum of the sample without bias.

PL is observed. This effect is caused by the electric-currentinduced radiative process, and is discussed elsewhere. ${ }^{37}$

\section{B. Physical mechanism}

Based on the presented data, we now discuss the physical mechanism responsible for the observed phonon resonances. Generally, a few processes are possible - resonant Raman scattering, phonon-assisted electron-hole recombination, phonon-assisted absorption, and phonon-assisted relaxation in the presence of nonradiative losses.

Resonant Raman scattering has a low efficiency, as observed, for example, by Sirenko et al. for a heterostructure with InP QD's. ${ }^{38}$ Our experimental results, namely, the dependence of the phonon resonance intensities on the applied bias and the strongly variable ratio of the $1 \mathrm{LO}$ and $2 \mathrm{LO}$ resonance intensities depending on spectral position within the PL band, allow us to rule out resonant Raman scattering as the process responsible for the phonon resonances. This point is further supported by the long decay time of the QD emission at the phonon resonances and the strong temperature dependence of the resonance intensities, discussed below.

Phonon-assisted electron-hole recombination can be also ruled out, because the electron-phonon interaction is weak in these structures and is able to produce only very small phonon sidebands in the PL spectra. ${ }^{39}$ In addition, the ratio of intensities of the one- and two-phonon sidebands must be almost independent of the photon energy of the excitation, in contrast to the observed behavior of the $1 \mathrm{LO}$ and $2 \mathrm{LO}$ resonances.

The phonon resonances in the spectra can also be caused by phonon-assisted absorption when an absorbed photon cre- ates an electron and a hole in their ground states, and a phonon. The probability of this process is usually equal to that of the phonon-assisted PL, and is also small. It can be enhanced by a resonance with some direct (phononless) optical transition as is discussed in Ref. 40. The phononassisted absorption in the $\mathrm{In}_{0.4} \mathrm{Ga}_{0.6}$ As QD's was observed by Findeis et ll $^{41}$ We neglect this process in the following discussion.

We assume that the main process causing phonon resonances is the selective phonon-assisted relaxation of hot carriers in the presence of nonradiative losses. The appearance of phonon resonances due to this process can be explained as follows. ${ }^{14}$ The quasiresonant excitation creates electrons and holes in the excited states. The QD's in the ensemble have slightly different sizes and shapes, and the interlevel energy spacing $\Delta E$ has some distribution. The spacing $\Delta E$ can well match the LO-phonon energy $E_{\mathrm{LO}}$ only in some subset of the QD's. Carrier relaxation in these QD's is fast due to the high efficiency of this process with the emission of a LO phonon. The relaxation in the rest of the QD's occurs via emission of acoustic phonons i.e., much more slowly. If the electron or hole can efficiently leave the QD (this process is usually referred to as nonradiative losses) before relaxation via acoustic-phonon emission, the PL does not appear in any spectral point except the point where it is "saved" by fast LO-phonon-assisted relaxation. In the time-integrated PL spectrum, a narrow peak shifted from the excitation line by the energy of the LO phonon, $E_{\mathrm{LO}}$, must be observed in this case. However, if there are no significant nonradiative losses, electrons and holes in any QD eventually relax to the lowest levels and recombine. In this case, the PL spectrum must reproduce the energy distribution of the lowest optical transition which usually has a bell-like smooth shape. Behavior of the spectra presented in Figs. 2 and 3 agrees with this scenario.

\section{Model}

The dependence of the PL spectra on the electric field was qualitatively discussed in Ref. 23. Here we analyze this dependence quantitatively in the framework of the model schematically drawn in Fig. 4. We assume that the quasiresonance excitation generates an electron and a hole in excited energy levels labeled by $|1 e\rangle$ and $|1 h\rangle$, respectively.

The excited electron and hole may relax at different rates. However, the experimental data do not allow us to separate these processes. On the other hand, the observed narrow resonances (see Figs. 2 and 3) can be formed only by onestep relaxation of carriers, with the emission of a single phonon. This may be relaxation of one of the photocreated carriers with another one being created in its ground state, ${ }^{42}$ or relaxation of an electron-hole pair as a correlated system. Therefore, we consider some effective relaxation rate from the excited state of the electron-hole pair to its ground state.

The relaxation process competes with the nonradiative losses. We assume that the external electric field activates the tunneling of the photocreated carriers from the QD into the barrier layer. The depth of the potential well for holes is usually smaller than that for electrons. As for InP QD's, it is 


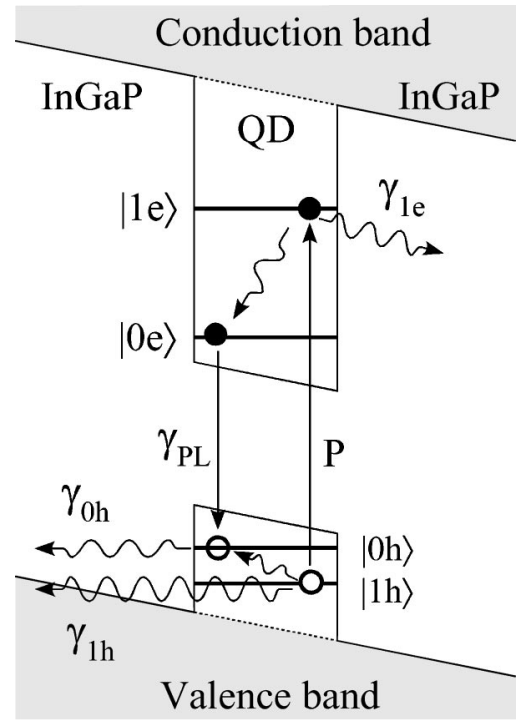

FIG. 4. A model of the PL quenching in the electric field. The vertical arrows marked by $P$ and $\gamma_{\mathrm{PL}}$ denote the pumping and radiative recombination processes, respectively. The relaxation of the hot carriers to their ground states is shown by the wavy arrows which are not labeled. The wavy arrows marked by $\gamma_{0 h}, \gamma_{1 h}$, and $\gamma_{1 e}$ indicate tunneling of the hole from the ground and excited states, and of the electron from the excited state, respectively.

assumed that the holes are weakly localized in the dots or even in the surrounding material. ${ }^{43,44} \mathrm{~A}$ real potential profile for carriers may be complicated due to strain and also due to composition fluctuations. ${ }^{45}$ Nevertheless we assume for simplicity that the potential barrier for holes acquires a triangular shape in the electric field, as shown in Fig. 4.

In the semiclassical approach, the tunneling rate $\gamma_{i}(U)$ from the state $|i\rangle$ through a triangular barrier is given by the expression $^{46}$

$$
\gamma_{i}=\gamma_{i}^{0} e^{-U_{i} / U}
$$

with

$$
U_{i}=(4 / 3 e \hbar) \sqrt{2 m^{*}} E_{i}^{3 / 2} d,
$$

where $m^{*}$ is the effective mass of a carrier, $E_{i}$ is the depth of the potential well for the carrier in state $|i\rangle$, and $d$ is the effective thickness of the insulating layer to which the bias is applied $(d \approx 0.5 \mu)$. Here we neglect the dependence of $E_{i}$ on $U$.

The well depth for electrons in the InP QD's is of about $200 \mathrm{meV}$, which prevents the electron from tunneling into the conduction band of the barrier layer at a moderate bias. However, the electrons may tunnel to deep levels that are present in the vicinity of the QD's even in high-quality heterostructures. ${ }^{37,47,48}$ Analysis shows that the electron tunneling from the excited state $|1 e\rangle$ affects the PL of the InP QD's at $U_{\text {bias }}<-1 \mathrm{~V}$. To simplify our discussion, we describe the rate of this process, $\gamma_{1 e}$, by the same equations (1). We also neglect variations of the carrier relaxation and optical transition probabilities in the electric field, a possible built-in electric field, ${ }^{47}$ and intrinsic dipole moment of
QD's. ${ }^{45}$ All these effects influence the PL of the QD's much more weakly than the carrier tunneling.

The dynamics of the electron-hole pair populations $n_{1}$ and $n_{0}$ in the levels $|1\rangle$ and $|0\rangle$, respectively, are described in the framework of the model by the equations

$$
\begin{gathered}
\frac{d n_{1}}{d t}=P-\left(\gamma_{r}+\gamma_{1 t}\right) n_{1}, \\
\frac{d n_{0}}{d t}=\gamma_{r} n_{1}-\left(\gamma_{\mathrm{PL}}+\gamma_{0 t}\right) n_{0} .
\end{gathered}
$$

Here $\gamma_{\mathrm{PL}}$ is the rate of the electron-hole radiative recombination, $\gamma_{r}$ is the relaxation rate from state $|1\rangle$ to state $|0\rangle$, and $\gamma_{1 t}=\gamma_{1 h}+\gamma_{1 e}$ and $\gamma_{0 t}=\gamma_{0 h}$ are the tunneling rates from the states $|1\rangle$ and $|0\rangle$, respectively.

For a cw excitation $(P=$ const $)$, the steady-state solution of Eq. (2) yields an expression for the bias dependence of the population of the radiative state $n_{0}$. As a result, the bias dependence of the PL intensity, which is proportional to $\gamma_{\mathrm{PL}} n_{0}$, is given by

$$
I_{\mathrm{PL}}(U)=N P \frac{1}{\left(1+\gamma_{1 t} / \gamma_{r}\right)} \frac{1}{\left(1+\gamma_{0 t} / \gamma_{\mathrm{PL}}\right)},
$$

where $N$ is the number of QD's which can emit light at a given spectral point under a given excitation.

The tunneling rates are equal to zero for zero bias [see Eqs. (1a) and (1b)]. Therefore, the PL intensity does not depend on the relaxation rate. This is why phonon resonances are not observed in the PL spectra in the absence of the electric field. In the presence of an electric field, Eq. (3) depends on the branching ratio of the relaxation and tunneling rates from the excited state $|1\rangle$. For slow acousticphonon relaxation, this ratio is larger and, therefore, the PL quenching is stronger than that for the fast LO-phonon relaxation.

To illustrate this behavior, in Fig. 5 we plot the bias dependence of the PL intensities for the sample with InP QD's at the LO resonance and also at the neighboring spectral point marked by $2 \mathrm{AC}$ in the inset of Fig. 5. The PL in the $2 \mathrm{AC}$ spectral point is contributed by those QD's in which the carriers have to relax with the emission of a few phonons because of the energy gap between the acoustic and LO phonons. The intensities $I_{\mathrm{LO}}(U)$ and $I_{2 \mathrm{AC}}(U)$ at the $\mathrm{LO}$ and $2 \mathrm{AC}$ spectral points, respectively, are fitted by Eq. (3). The ratio of these intensities, normalized to unity at zero bias, may be used as a measure of the contrast of the features in the PL spectrum at strong bias. The bias dependence of this ratio is plotted in Fig. 5, and fitted by the equation

$$
\frac{I_{\mathrm{LO}}(U)}{I_{2 \mathrm{AC}}(U)}=\frac{\left(1+\gamma_{1 t} / \gamma_{2 \mathrm{AC}}\right)}{\left(1+\gamma_{1 t} / \gamma_{\mathrm{LO}}\right)},
$$

that follows directly from Eq. (3). Here $\gamma_{\mathrm{LO}}$ and $\gamma_{2 \mathrm{AC}}$ are relaxation rates with the emission of a $\mathrm{LO}$ phonon and two acoustic phonons, respectively. As is seen from the figure, the calculated curves reproduce the experimental values reasonably well. Thus, in spite of its simplicity, the model ad- 


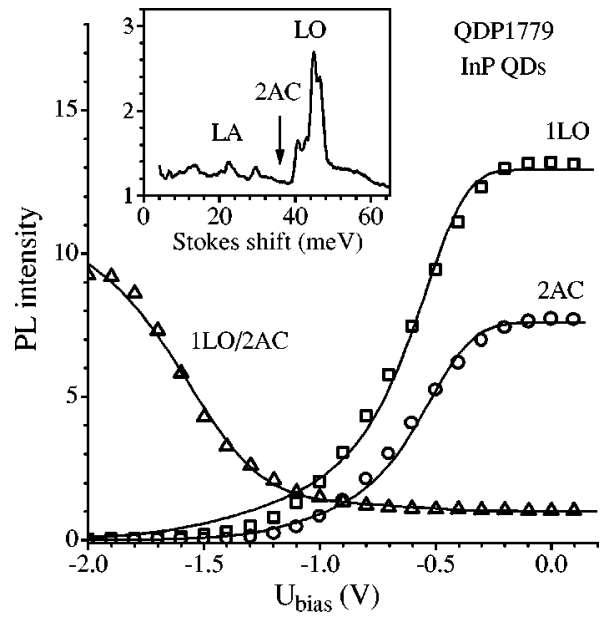

FIG. 5. Bias dependence of the PL intensities at the LO (squares) and 2AC (circles) spectral points and of their normalized ratio (triangles) for the sample with InP QD's. $E_{\text {exc }}=1771 \mathrm{meV}$. Solid curves the fits by Eqs. (3) and (4). The fitting parameters for all the curves are $U_{1 h}=2 \mathrm{~V}, U_{0 h}=4 \mathrm{~V}, U_{1 e}=16 \mathrm{~V}, \gamma_{1 e}^{0} / \gamma_{1 h}^{0}$ $=3.8 \times 10^{4}, \gamma_{0 h}^{0} / \gamma_{\mathrm{PL}}=150, \gamma_{\mathrm{LO}} / \gamma_{2 \mathrm{AC}}=12$, and $\gamma_{1 h}^{0} / \gamma_{\mathrm{LO}}=0.2$. Inset: the PL spectrum at $U_{\text {bias }}=-1.5 \mathrm{~V}$.

equately describes, as a whole, the behavior of the PL intensity of the InP QD's versus the applied voltage.

From the fit, we have determined $U_{1 h}=2 \mathrm{~V}, U_{0 h}$ $=4 \mathrm{~V}$, and $U_{1 e}=16 \mathrm{~V}$. These values allow one to estimate the depth of the potential wells for carriers by means of Eq. (1b). The values $E_{0 h}=13 \mathrm{meV}, E_{1 h}=9 \mathrm{meV}$, and $E_{1 e}$ $=65 \mathrm{meV}$ were obtained using the heavy-hole effective mass, ${ }^{49} m_{h}^{*}=0.65 m_{0}$, and the electron effective mass, $m_{e}^{*}$ $=0.08 m_{0}$, in the InP crystal. ${ }^{50}$ Here $m_{0}$ is the electron mass. The obtained results show that the potential well for holes in InP QD's is small and positive, i.e., the holes are localized in the dots. This conclusion is further supported by the relatively high probability of optical transitions in the InP QD's, as discussed in Sec. III D.

The accuracy of the values of $E_{0 h}$ and $E_{1 h}$ is limited by many simplifications used in the model. We estimate an accuracy of about $50 \%$ by analysis of all sets of experimental data, as well as by comparing the results using various modifications of the described model. We would like to stress that the actual accuracy can be verified only by independent measurements of these values with a different technique.

We performed a similar analysis of the PL data for $\mathrm{In}_{0.35} \mathrm{Ga}_{0.65} \mathrm{As}$ QD's presented in Fig. 3. The bias dependence of the $\mathrm{LO}_{\mathrm{I}}$ and LA resonances is shown in Fig. 6. Both dependencies are well fitted by the equation

$$
I_{\mathrm{PL}}(U)=P /\left(1+\gamma_{0 t} / \gamma_{\mathrm{PL}}\right)
$$

accounting only for a tunneling from the radiative state $|0\rangle$. This means that tunneling from the excited state $|1\rangle$ at values of the bias used in our experiment is much slower than carrier relaxation with emission of $\mathrm{LO}_{\mathrm{I}}$ and LA phonons. Assuming that the hole tunneling process is responsible for PL quenching, ${ }^{51}$ from the fit we can obtain the energy of the lowest hole state $E_{0 h}=70 \mathrm{meV}$. This value agrees with typical values considered for $\operatorname{In}_{0.35} \mathrm{Ga}_{0.65}$ As QD's. ${ }^{4}$

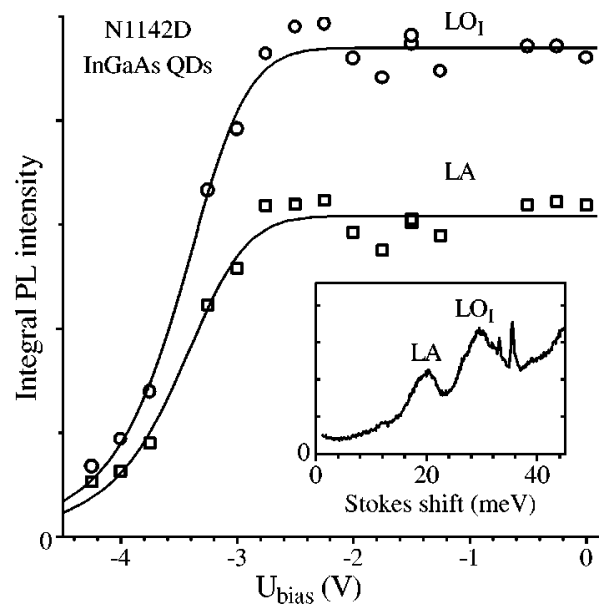

FIG. 6. Bias dependence of the PL intensity at the $\mathrm{LO}_{\mathrm{I}}$ (circles) and LA (squares) spectral points for the sample with $\operatorname{In}_{0.35} \mathrm{Ga}_{0.65} \mathrm{As}$ QD's. The data are taken from the spectra presented in Fig. 3. Solid curves are fits by Eq. (3). The fitting parameters are $U_{0}=40 \mathrm{~V}$ and $\gamma_{0}^{0} / \gamma_{\mathrm{PL}}=9 \times 10^{4}$. Inset: PL spectrum at $U_{\text {bias }}=-3 \mathrm{~V}$.

\section{Kinetics}

Kinetic measurements of PL allow one to study the time evolution of the radiative state population $n_{0}$. The time resolution of our setup (6 ps) was enough to study the relaxation of the photocreated carrier in real time. We also performed a further verification of the model proposed above by analyzing the kinetics in the electric field.

Examples of the PL kinetics under quasiresonant excitation for two spectral points within the PL band are shown in Fig. 7. The kinetics consists of a relatively short rising part and a rather long decay. The PL leading edge is shorter than the time resolution of the setup for LO resonance and much longer for other spectral points, as shown in the inset. At negative bias, the decay becomes faster and the amplitude of the signal becomes smaller.

The time evolution of the PL intensity can be described by an equation that follows from Eqs. (2). Assuming that the excitation pulse is shorter than any other process under consideration, $P=P_{0} \delta(t)$, we obtain

$$
I_{\mathrm{PL}}(t)=I_{0}\left\{e^{-\left(\gamma_{\mathrm{PL}}+\gamma_{0 t}\right) t}-e^{-\left(\gamma_{r}+\gamma_{1 t}\right) t}\right\},
$$

where

$$
I_{0}=\gamma_{r} P_{0} /\left[\left(\gamma_{r}-\gamma_{\mathrm{PL}}\right)+\left(\gamma_{1 t}-\gamma_{0 t}\right)\right]
$$

The two terms of the Eq. (6) describe the decay and rise of the PL pulse, respectively.

The function $I_{\mathrm{PL}}(t)$ given by Eq. (6) well fits the kinetics for the 2AC spectral point, as seen in Fig. 7. However, the behavior of the PL kinetics at LO resonance is more complicated. At zero bias, the fast and slow components of the PL rise are clearly seen. The kinetics is well fitted by

$$
I_{\mathrm{LO}}(t)=I_{0}\left\{e^{-\left(\gamma_{\mathrm{PL}}+\gamma_{0 t}\right) t}-\left[\alpha e^{-\gamma_{\mathrm{LO}} t}+(1-\alpha) e^{-\gamma_{\mathrm{ac}} t}\right] e^{-\gamma_{1 t} t}\right\} .
$$

The slow PL rise component is probably due to multistep carrier relaxation with emission of acoustic phonons in some 


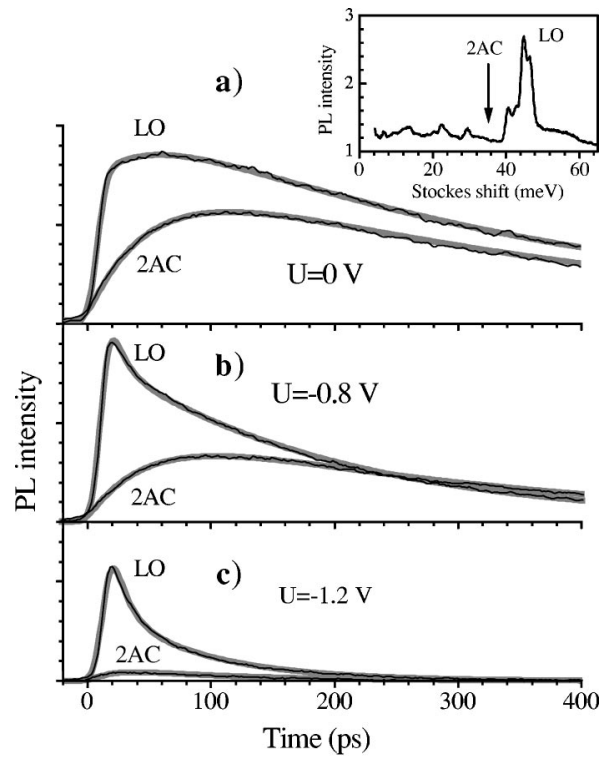

FIG. 7. PL kinetics of the sample with the InP QD's (thin solid line) for different biases at the LO and 2AC spectral points marked in the inset and a fit (thick gray line) by Eqs. (6) - (8) as discussed in the text. Values of the fitting parameters $\gamma_{\mathrm{PL}}+\gamma_{0 t}=\tau_{\mathrm{PL}}(U)^{-1}$ and $\gamma_{r}+\gamma_{1 t}=\tau_{r}(U)^{-1}$ for LO and 2AC kinetics, respectively, can be extracted from Fig. 8. Values of the other parameters for $2 \mathrm{AC} \mathrm{ki-}$ netics are $\gamma_{\mathrm{PL}}+\gamma_{0 t}=(358 \mathrm{ps})^{-1},(230 \mathrm{ps})^{-1}$, and $(104 \mathrm{ps})^{-1}$ for $U=0,-0.8$, and $-1.2 \mathrm{~V}$, respectively. Values of the other parameters for LO kinetics are $\gamma_{\mathrm{ac}}+\gamma_{1 t}=(43.3 \mathrm{ps})^{-1}$ and $\alpha=0.67$ for $U=0 \mathrm{~V}$, and $\gamma_{\mathrm{ac}}+\gamma_{0 t}=(9.9 \mathrm{ps})^{-1}$ and $\beta=0.60$ for $U=$ $-0.8 \mathrm{~V}$, and $\gamma_{\mathrm{ac}}+\gamma_{0 t}=(11.8 \mathrm{ps})^{-1}$ and $\beta=0.37$ for $U=$ $-1.2 \mathrm{~V}$. Values of the parameter $\gamma_{\mathrm{LO}}+\gamma_{1 t}$ is chosen to be $1 \mathrm{ps}^{-1}$ for all biases. The fitting curves are convoluted with Gaussian with a FWHM of 6 ps. $E_{\text {exc }}=1771 \mathrm{meV}$. Inset: the PL spectrum at $U_{\text {bias }}=-1.5 \mathrm{~V}$.

fraction $(1-\alpha)$ of the QD's. The carrier tunneling at negative bias compensates for this process, and another process causing a relatively fast PL decay component becomes observable [see Figs. 7(b) and 7(c)]. This process is probably the cascade relaxation of the carriers in "large", QD's in which the lowest-energy optical transition is redshifted relative to the given spectral point. In this case the PL kinetics is described by

$$
I_{\mathrm{LO}}(t)=I_{0}\left\{\left[\beta e^{-\gamma_{\mathrm{ac}} t}+(1-\beta) e^{-\gamma_{\mathrm{PL}} t}\right] e^{-\gamma_{0 t} t}-e^{-\left(\gamma_{\mathrm{LO}}+\gamma_{1 t}\right) t}\right\},
$$

where $\beta$ is the fraction of "large", QD's.

A good agreement of the fits with the PL kinetics allows one to determine the bias dependence of the PL decay time, $\tau_{\mathrm{PL}}(U)$, and the PL rise time, $\tau_{\mathrm{r}}(U)$. In Fig. 8 we show the bias dependencies of $\tau_{\mathrm{PL}}(U)$ for the LO resonance and of $\tau_{\mathrm{r}}(U)$ for the $2 \mathrm{AC}$ spectral point. According to Eqs. (6) $-(8)$, they are described by the equations

$$
\tau_{\mathrm{PL}}(U)=\frac{\tau_{\mathrm{PL}}}{1+\tau_{\mathrm{PL}} \gamma_{0 t}}, \quad \tau_{r}(U)=\frac{\tau_{r}}{1+\tau_{r} \gamma_{1 t}} .
$$

The data in Fig. 8 are fitted by these functions using Eq. (1) for the tunneling rate, $U_{0}=4 \mathrm{~V}$ determined above, and $U_{1}$
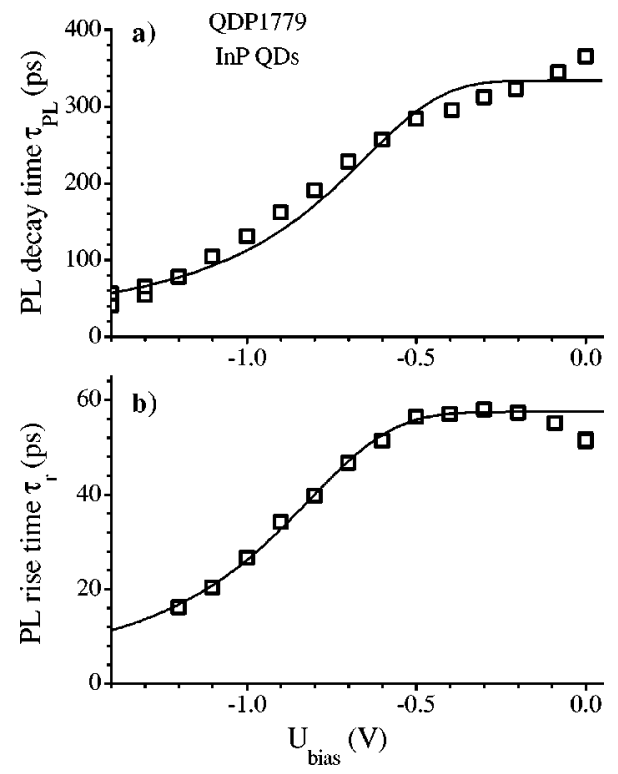

FIG. 8. Bias dependence of the PL decay time (a) and the PL rise time (b). Open squares represent the data obtained by fitting of the PL kinetics. Fits by Eqs. (9) are shown by solid lines. The fitting parameters are (a) $\tau_{\mathrm{PL}}=330 \mathrm{ps}, \gamma_{0 t}^{0}=0.2 \mathrm{ps}^{-1}$, and $U_{0}=4 \mathrm{~V}$; and (b) $\tau_{\mathrm{r}}=58 \mathrm{ps}, \gamma_{1 t}^{0}=2.5 \mathrm{ps}^{-1}$, and $U_{0}=5.5 \mathrm{~V}$.

as a fitting parameter. ${ }^{52}$ As seen, the fit with $U_{1}=5.5 \mathrm{~V}$ well reproduces general behavior of the rise and decay times.

As seen in Fig. 8(a), the PL decay time at small bias is about 300 ps. Experiments show that this time, as well as the PL intensity of the QD's, are almost independent of the sample temperature within several tens of $\mathrm{K}$. This is an indication that PL decay is determined by radiative recombination of the electron-hole pair. This means that nonradiative losses of excitation at small bias are negligibly small. A smooth profile of the PL band of the QD's observed at any excitation energies (see Fig. 1) provides additional evidence of this conclusion. The obtained value of $\tau_{\mathrm{PL}}$ also agrees well with the model calculations of the radiative life time for disk-shaped QD's. ${ }^{53}$ Thus we can speak about a relatively high probability of the optical transitions in InP QD's due to a good overlap of the electron and hole wave functions.

The PL rise time at the $2 \mathrm{AC}$ spectral point is about $60 \mathrm{ps}$ at small bias [see Fig. 8(b)]. Similar kinetic measurements at acoustic resonances give a PL rise time that is approximately twice as small. The PL rise time at the LO resonance is shorter than our time resolution of $6 \mathrm{ps}$. A lower limit for this time can be deduced from the FWHM of the separate peaks of the LO resonances. It is about $1.2 \mathrm{meV}$ for the InP QD's (see Fig. 2), that corresponds to a relaxation time of $0.6 \mathrm{ps}$. The corresponding value for $\operatorname{In}_{0.35} \mathrm{Ga}_{0.65} \mathrm{As}$ QD's is about $1.5 \mathrm{ps}$.

\section{E. Acoustic-phonon resonances}

The PL spectra of the biased samples as well as the PL kinetic data presented above demonstrate that the carrier relaxation with emission of high-energy acoustic phonons is efficient, in contradiction to theoretical predictions. ${ }^{5,7}$ To make this contradiction evident, in Fig. 9 we plot the PL 


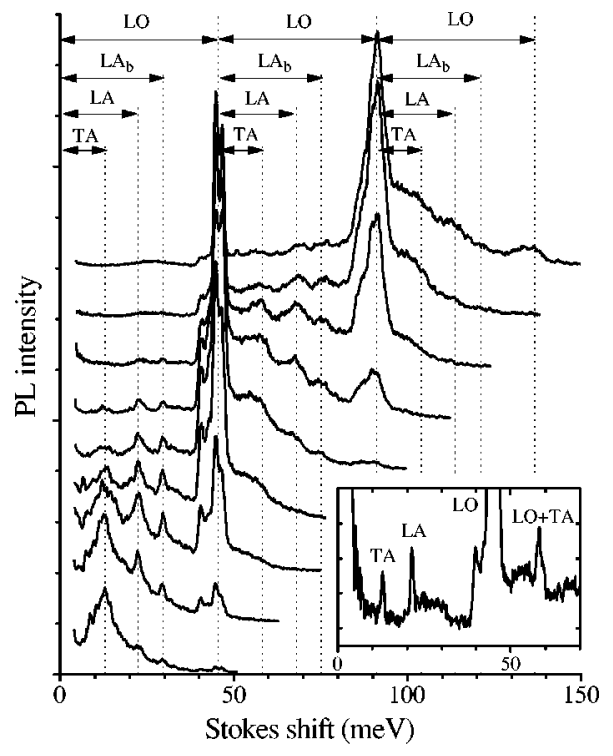

FIG. 9. The magnified PL spectra presented in Fig. 2 at different photon energies of excitation (from top to bottom) vs the Stokes shift. $U_{\text {bias }}=-1.5 \mathrm{~V}$. The spectra are shifted vertically for clarity. Similar phonon resonances in different spectra are marked by vertical lines. The arrows labelled 'TA', etc., indicate the energy shift of the resonances from the laser line or $1 \mathrm{LO}$ or $2 \mathrm{LO}$ resonances. Inset: the PL spectrum at $U_{\text {bias }}=-2 \mathrm{~V}$ vs the Stokes shift.

spectra presented in Fig. 2 versus the Stokes shift. It is seen that the various acoustic-phonon resonances are clearly observed not only between the laser line and the $1 \mathrm{LO}$ resonance, but also between the $1 \mathrm{LO}$ and $2 \mathrm{LO}$ resonances.

We cannot propose any quantitative description of the phenomenon, but there are a few points that, in our opinion, can be important for future modeling. The observed phonon resonances are caused by high-energy acoustic phonons. Acoustic phonons with such energy in the bulk crystal have a large momentum near the Brillouin zone boundary. This also holds with good accuracy for structures with QD's, because the confinement effect for the acoustic phonons in these samples may be considered weak due to the small difference between the elastic properties of the QD and the barrier material.

The interaction of a carrier with phonons of large momentum is efficient if the energy state of the carrier is characterized by the same momentum. The energy states of carriers in a QD are formed by a mixture of bulk states due to confinement. In other words, the wave function of the quantumconfined state in a QD is a linear combination of wave functions of various states in the Brillouin zone. To explain the observed resonances, we have to assume that this linear combination contains a considerable contribution from the large momentum states such as, for example, the states of the $L$ valley.

To support this assumption, we would like to note that the energy shift of the lowest electronic state in InP and $\mathrm{In}_{0.35} \mathrm{Ga}_{0.65} \mathrm{As}$ QD's due to confinement is about a few hundred meV. This value is comparable with the energy separations of the minima in the $\Gamma$ and $L$ or $X$ valleys in these materials. ${ }^{50}$ It should also be taken into account that the ef- fective mass of electrons in valleys near the Brillouin zone boundary is large, and, hence, the energy shift of the states due to confinement is much smaller than that at the $\Gamma$ point. As a result, the energy separation between states of the $\Gamma$ and $L$ or $X$ valleys is decreased with the decreasing size of the QD. This may be the reason why the mixing of these states in the QD's is not small. A significant decrease of the energy separation between the $\Gamma$ and $L$ minima in InP QD's was observed by Menoni et al. ${ }^{54}$ This decrease can also be induced by strain due to lattice mismatch of the QD's and surrounding material. An observation of the $\Gamma-X$ crossover under high pressure in the InP QD's (Ref. 55) and in the InAs QD's (Ref. 56) supports this assumption.

To model this situation it is necessary to go beyond the widely used effective-mass approximation that constructs QD energy states from Bloch functions in the neighborhood of the $\Gamma$ point. In fact, empirical pseudopotential calculations already showed the importance of $\Gamma-X-L$ valley mixing in QD's (see, for example, Ref. 57). Similar calculations for the structures studied in this work would be of interest.

Some experimental evidence of the above assumption seems to be provided by the spectrum shown in the inset of Fig. 9, where the TA, LA, and LO+TA resonances look like narrow peaks with a FWHM of about 2-3 meV. The formation of such narrow peaks is possible only if a small portion of the acoustic phonons causes a sufficiently strong relaxation. According to the above assumption, a selection of these acoustic phonons is caused by the wave-vector selection rule. It should be also taken into account that phonons with wave numbers near the Brillouin-zone boundary show small dispersions.

The interaction with the high-energy acoustic phonons may be also enhanced for two other reasons. The first is the small group velocity of the phonons that lengthens the interaction time. The second is the piezoelectric effect in these crystals that leads to long-range electric fields associated with acoustic phonons. Modeling these interactions at a large wave vector $q$ of phonons is the open problem.

\section{F. Auger-like processes}

The phonon resonances discussed above can be observed under specific experimental conditions. The most important conditions are low PL efficiency, low power density of excitation, and low temperature. We can consider the data presented above as a illustrations of the resonances arising due to a decrease of the PL efficiency. In this section, we consider the disappearance of the resonances with increasing pump power.

We have measured the pump power dependence of the PL spectra and the kinetics of the biased sample QDP1779 with the InP QD's. 5-ps pulses, with a repetition period of about $12 \mathrm{~ns}$, were used to increase the peak power of the excitation. The measured spectra are shown in Fig. 10. As seen, the acoustic-phonon resonances disappear and the LO resonance becomes barely observable under strong pumping. This fact means that the relaxation rate for all interlevel spacings becomes comparable to the LO-phonon-assisted relaxation rate. The PL kinetics reveals a steplike component of the PL rise 


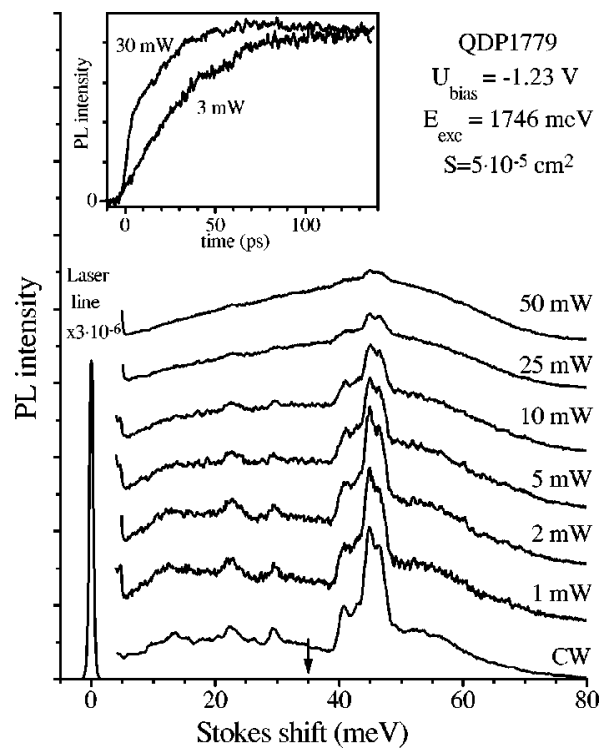

FIG. 10. PL spectra of the biased sample with InP QD's under cw excitation (bottom curve) and pulsed excitation with average power indicated against each curve. The laser spot area on the sample is $S=5 \times 10^{-5} \mathrm{~cm}^{2} ; \quad E_{\text {exc }}=1746 \mathrm{meV}$ and $U_{\text {bias }}=$ $-1.23 \mathrm{~V}$. An arrow marks the $2 \mathrm{AC}$ spectral point where the PL kinetics was measured. Inset: the PL kinetics under weak and strong excitations normalized at $130 \mathrm{ps}$.

with an increase of the pumping (see the inset of Fig. 10), which also indicates an acceleration of the carrier relaxation.

The observed behavior of the PL spectra and kinetics can be explained by invoking Auger-like processes. The Augerlike process involved here is carrier-carrier scattering, in which one carrier loses its energy and the other one acquires it. ${ }^{8,11,30-32}$ On the one hand, relaxation due to Auger-like processes should be fast because the carriers as charged particles interact with each other much more strongly than with phonons. On the other hand, this process is possible if separation between the energy levels for the first carrier coincides with that for the second one. In the case of the InP QD's, this condition can be easily fulfilled for electron-hole or holehole scattering, because of the large density of the high energy levels for holes. Also, the hole could be ejected out of the QD into the continuum of the barrier layer, so that the energy conservation condition is easily fulfilled. ${ }^{30,31}$ It is evident that we should not expect any spectral features (resonances) due to relaxation via an Auger-like process. It is also evident that the efficiency of this process should depend on the number of carriers in the QD, because each carrier can be scattered by any other one. In the simplest model, the dependence on the pump power should be quadratic.

These features of the Auger-like process agree qualitatively with the experiment. Therefore, we can conclude that a strong pumping activates an alternative relaxation mechanism that is much faster than the acoustic-phonon-assisted relaxation. The Auger relaxation rate achieved in our experiments is comparable with the rate of the LO-phonon-assisted relaxation, that is about $1 \mathrm{ps}^{-1}$.

A quantitative analysis of the presented data is difficult because the absorption coefficient for the InP QD's at the

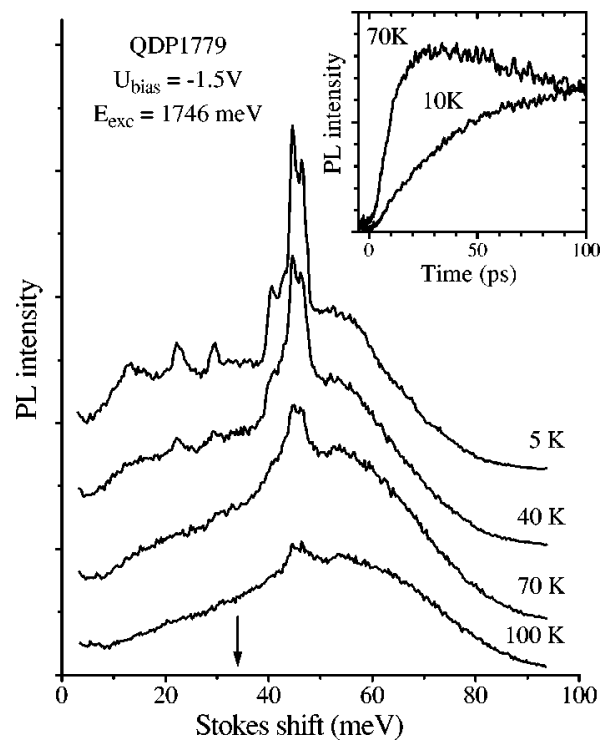

FIG. 11. PL spectra of the biased sample with InP QD's at different temperatures indicated near each spectrum. $E_{\text {exc }}$ $=1746 \mathrm{meV}$ and $U_{\text {bias }}=-1.5 \mathrm{~V}$. The arrow indicates the spectral point at which the PL kinetics were measured. Inset: the normalized PL kinetics at temperatures indicated for each curve.

laser line is unknown. ${ }^{58}$ As a result, the number of photocreated electron-hole pairs in a QD cannot be estimated correctly. In addition, the observed pump power dependence of the PL intensity is more complicated than quadratic. The reason for this is probably some additional processes at strong pumping such as saturation of absorption that should lead to sublinear dependence, or saturation of deep levels that should lead to superlinear dependence.

\section{G. Thermostimulated relaxation}

Another way to accelerate the carrier relaxation is to increase the sample temperature. Figure 11 depicts the PL spectra of the biased sample at a few elevated temperatures. It is clearly seen that phonon resonances become smaller relative to the structureless pedestal in the spectrum at $T$ $=100 \mathrm{~K}$. The temperature increase at first destroys the lowest in energy phonon resonance (TA), then the other acoustic resonances, and finally the $\mathrm{LO}$ resonance.

This behavior is easy to understand. Heating of the sample creates phonons. These phonons can cause carrier relaxation due to stimulated emission of phonons into the same phonon modes. The number of phonons with small energy is always larger then that with high energy. Therefore, the stimulated emission is more efficient for low-energy phonons. The PL kinetics shown in the inset of Fig. 11 also demonstrates the shortening of the carrier relaxation time.

An important result is that the rate of relaxation at about $100 \mathrm{~K}$ becomes comparable to that of the LO-phononassisted relaxation, independent of the spectral point. Thus we can conclude that no retarded relaxation of the hot carriers in QD's should be expected at elevated temperatures. This result is important for device applications. 


\section{CONCLUSION}

The present study of PL spectra of InP and $\operatorname{In}_{0.35} \mathrm{Ga}_{0.65} \mathrm{As}$ QD's in an electric field allowed us to establish the nature of the observed narrow resonances. These are shown to arise due to a competition of the fast one-step relaxation of hot carriers with emission of an acoustic or LO phonon and nonradiative losses. For InP and $\mathrm{In}_{0.35} \mathrm{Ga}_{0.65}$ As QD's in an electric field, the nonradiative losses are mainly caused by hole tunneling from the QD's into the barrier layer.

We developed a model that quantitatively describes the bias dependence of PL intensity at the phonon resonances. This model allowed us to estimate the depth of the potential wells for holes in the studied heterostructures.

The proposed model was verified by the PL kinetic measurements for the samples with InP QD's. We found that the carrier relaxation is really fast with emission of not only LO phonons but also acoustic phonons. This result gives clear evidence of the relatively strong carrier-acoustic-phonon coupling in the QD's. We assume the main reason for this is a mixing of $\Gamma$ electronic states with the states with large

*Electronic address: ivan@paloma.spbu.ru

†Permanent address: Vavilov State Optical Institute, St.-Petersburg, Russia.

${ }^{\ddagger}$ Present address: Space Vacuum Epitaxy Center, University of Houston, Houston, Texas 77204-5507.

${ }^{1}$ D. Gammon, Science 280, 225 (1998).

${ }^{2}$ Y. Arakawa and A. Yariv, IEEE J. Quantum Electron. QE-22, 1887 (1986).

${ }^{3}$ D. Bimberg, M. Grundmann, and N. N. Ledenstov, Quantum Dot Heterostructures (Wiley, New York, 1999).

${ }^{4}$ M. Sugawara, Self-Assembled InGaAs/GaAs Quantum Dots Semiconductors and Semimetals (Academic Press, New York, 1999), Vol. 60.

${ }^{5}$ U. Bockelmann and G. Bastard, Phys. Rev. B 42, 8947 (1990).

${ }^{6}$ H. Benisty, C. M. Sotomayor-Torrès, and C. Weisbuch, Phys. Rev. B 44, 10945 (1991).

${ }^{7}$ T. Inoshita and H. Sakaki, Phys. Rev. B 46, 7260 (1992).

${ }^{8}$ U. Bockelmann and T. Egeler, Phys. Rev. B 46, 15574 (1992).

${ }^{9}$ U. Bockelmann, Phys. Rev. B 48, 17637 (1993).

${ }^{10}$ H. Benisty, Phys. Rev. B 51, 13281 (1995).

${ }^{11}$ A. L. Efros, V. A. Kharchenko, and M. Rosen, Solid State Commun. 93, 281 (1995).

${ }^{12}$ P. C. Sercel, Phys. Rev. B 51, 14532 (1995).

${ }^{13}$ S. Fafard, R. Leon, D. Leonard, J. L. Merz, and P. M. Petroff, Phys. Rev. B 52, 5752 (1995).

${ }^{14}$ R. Heitz, M. Grundmann, N. N. Ledentsov, L. Eckey, M. Veit, D. Bimberg, V. M. Ustinov, A. Yu. Egorov, A. E. Zhukov, P. S. Kop'ev, and Zh. I. Alferov, Appl. Phys. Lett. 68, 361 (1996).

${ }^{15}$ K. Mukai, N. Ohtsuka, H. Shoji, and M. Sugawara, Phys. Rev. B 54, R5243 (1996).

${ }^{16}$ K. H. Schmidt, G. Medeiros-Ribeiro, M. Oestreich, P. M. Petroff, and G. H. Döhler, Phys. Rev. B 54, 11346 (1996).

${ }^{17}$ B. Ohnesorge, M. Albrecht, J. Oshinowo, A. Forchel, and Y. Arakawa, Phys. Rev. B 54, 11532 (1996).

${ }^{18}$ M. Vollmer, E. J. Mayer, W. W. Rühle, A. Kurtenbach, and K. Eberl, Phys. Rev. B 54, 17292 (1996). momentum due to confinement of the electron in a QD.

We have also studied carrier relaxation in the presence of more than one electron-hole pair in a QD. Clear evidence is found for carrier-carrier scattering giving rise to acceleration of the relaxation. A similar effect was observed under heating of the sample where the acceleration of the relaxation is caused by stimulated phonon emission.

By careful analysis of the experimental data, we conclude that there is no phonon bottleneck effect in QD's, at least in the sense proposed in Refs. 5 and 6. Carrier relaxation with emission of acoustic or LO phonons is much faster than radiative recombination. Our experiments clearly show that phonon-assisted relaxation is the main channel for the carrier relaxation at low power density.

\section{ACKNOWLEDGMENTS}

The authors wish to thank Dr. J. Qi and Dr. K. Nishi for sample preparation, and Dr. I. Gerlovin and Dr. V. Zapasskii for a fruitful discussion.

${ }^{19}$ M. J. Steer, D. J. Mowbray, W. R. Tribe, M. S. Skolnick, M. D. Sturge, M. Hopkinson, A. G. Cullis, C. R. Whitehouse, and R. Murray, Phys. Rev. B 54, 17738 (1996).

${ }^{20}$ R. Heitz, M. Veit, N. N. Ledentsov, A. Hoffmann, D. Bimberg, V. M. Ustinov, P. S. Kop'ev, and Zh. I. Alferov, Phys. Rev. B 56, 10435 (1997).

${ }^{21}$ X. Q. Li, H. Nakayama, and Y. Arakawa, Phys. Rev. B 59, 5069 (1999).

${ }^{22}$ Y. Toda, O. Moriwaki, M. Nishioka, and Y. Arakawa, Phys. Rev. Lett. 82, 4114 (1999).

${ }^{23}$ I. V. Ignatiev, I. E. Kozin, S. V. Nair, H.-W. Ren, S. Sugou, and Y. Masumoto, Phys. Rev. B 61, 15633 (2000).

${ }^{24}$ V. F. Gandmacher and Y. B. Levinson, Carrier Scattering in Metals and Semiconductors (North-Holland, Amsterdam, 1987).

${ }^{25}$ M. Grundmann, R. Heitz, N. Ledentsov, O. Stier, D. Bimberg, V. M. Ustinov, P. S. Kop'ev, Zh. I. Alferov, S. S. Ruvimov, P. Werner, U. Gosele, and J. Heydenreich, Superlattice. Microstruct. 19, 81 (1996).

${ }^{26}$ C. Guasch, C. M. Sotomayor-Torrès, N. N. Ledentsov, D. Bimberg, V. M. Ustinov, and P. S. Kop'ev, Superlattices. Microstruct. 21, 509 (1997).

${ }^{27}$ A. V. Baranov, V. Davydov, H.-W. Ren, S. Sugou, and Y. Masumoto, J. Lumin. 87-89, 503 (2000).

${ }^{28}$ D. F. Schroeter, D. J. Griffiths, and P. C. Sercel, Phys. Rev. B 54, 1486 (1996).

${ }^{29}$ X.-Q. Li and Y. Arakawa, Phys. Rev. B 56, 10423 (1997).

${ }^{30}$ M. Braskén, M. Lindberg, M. Sopanen, H. Lipsanen, and J. Tulkki, Phys. Rev. B 58, R15993 (1998).

${ }^{31}$ S. Nair and Y. Masumoto, J. Lumin. 87-89, 408 (2000); Phys. Status Solidi B 178, 303 (2000).

${ }^{32}$ R. Ferreira and G. Bastard, Appl. Phys. Lett. 74, 2818 (1999).

${ }^{33}$ F. Clérot, B. Deveaud, A. Chomette, A. Regreny, and B. Sermage, Phys. Rev. B 41, 5756 (1990).

${ }^{34}$ K. H. Schmidt, G. Medeiros-Ribeiro, and P. M. Petroff, Phys. Rev. B 58, 3597 (1998).

${ }^{35}$ I. E. Kozin, I. V. Ignatiev, H.-W. Ren, S. Sugou, and Y. Masu- 
moto, J. Lumin. 87-89, 441 (2000).

${ }^{36}$ E. Bedel, G. Landa, R. Charles, J. P. Redouleś, and J. B. Renussi, J. Phys. C 19, 1471 (1986).

${ }^{37}$ I. Ignatiev, I. Kozin, H.-W. Ren, S. Sugou, and Y. Masumoto, Phys. Rev. B 60, R14001 (1999).

${ }^{38}$ A. A. Sirenko, M. K. Zundel, T. Ruf, K. Eberl, and M. Cardona, Phys. Rev. B 58, 12633 (1998).

${ }^{39}$ R. Heitz, I. Mukhametzhanov, O. Stier, A. Madhukar, and D. Bimberg, Phys. Rev. Lett. 83, 4654 (1999).

${ }^{40}$ V. Davydov, I. V. Ignatiev, I. E. Kozin, S. V. Nair, J.-S. Lee, H.-W. Ren, S. Sugou, and Y. Masumoto, Phys. Status Solidi B (to be published).

${ }^{41}$ F. Findeis, A. Zrenner, G. Bohm, and G. Abstreiter, Phys. Rev. B 61, R10579 (2000).

${ }^{42}$ Optical transitions between the electron and hole states with different quantum numbers, e.g., $|0 h\rangle$ and $|1 e\rangle$, are forbidden only for highly symmetrical QD's. We cannot exclude these transitions from consideration for the studied QD's because of their low symmetry.

${ }^{43}$ C. Pryor, M.-E. Pistol, and L. Samuelson, Phys. Rev. B 56, 10404 (1996).

${ }^{44}$ M. Hayne, R. Provoost, M. K. Zundel, Y. M. Manz, K. Eberl, and V. V. Moshchalkov, Phys. Rev. B 62, 10324 (2000).

${ }^{45}$ P. W. Fry, I. E. Itskevich, D. J. Mowbray, M. S. Skolnick, J. J. Finley, J. A. Barker, E. P. O’Reilly, L. R. Wilson, L. A. Larkin, P. A. Maksym, M. Hopkinson, M. Al-Khafaji, J. P. R. David, A. G. Gullis, G. Hill, and J. C. Clark, Phys. Rev. Lett. 84, 733 (2000)

${ }^{46}$ L. D. Landau and E. M. Lifshitz, Quantum Mechanics, 3rd ed (Pergamon, Oxford, 1977), Sec. 50.

${ }^{47}$ V. Davydov, I. Ignatiev, H.-W. Ren, S. Sugou, and Y. Masumoto, Appl. Phys. Lett. 74, 3002 (1999).

${ }^{48}$ P. C. Sercel, Al. L. Efros, and M. Rosen, Phys. Rev. Lett. 83, 2394 (1999).

${ }^{49}$ Size quantization can mix the heavy- and light-hole bands, which results in a change of the effective mass. This mixing leads to some uncertainty in the mass that should be used in the model. However the energies $E_{i}$, calculated using Eq. (1b) depends weakly (as $m^{-1 / 3}$ ) on the mass and cannot differ by more than
$30 \%$ from the values given in the text.

${ }^{50}$ Semiconductors, Intrinsic Properties of Group V Elements and III-V, II-VI, and I-VII Compounds, edited by K.-H. Hellwege and O. Madelung, Landolt-Börnstein, New Series, Group III, Vol. 22, Pt. a (Springer-Verlag, Berlin, 1987), pp. 120, 191, and 351.

${ }^{51} \mathrm{We}$ used the heavy-hole effective mass $m_{h h}=0.43 m_{0}$. Actually, we cannot uniquely determine the type of carrier, electron or hole, which tunnels from the QD. In the case of electron tunneling, a similar estimation using the electron mass $m_{e}=0.05 m_{0}$ gives rise to the energy of the lowest electron state $E_{0 e}$ $=140 \mathrm{meV}$. This value can also be reasonable for the studied sample.

${ }^{52}$ We used a simple fit for the data in Fig. 8(b) because of limited time resolution.

${ }^{53}$ M. Sugawara, Phys. Rev. B 51, 10743 (1995).

${ }^{54}$ C. S. Menoni, L. Miao, D. Patel, O. I. Mic'ic', and A. J. Nozik, Phys. Rev. Lett. 84, 4168 (2000).

${ }^{55}$ C. Ulrich, S. Ves, A. R. Goñi, A. Kurtenbach, K. Syassen, and K. Eberl, Phys. Rev. B 52, 12212 (1995).

${ }^{56}$ I. E. Itskevich, M. S. Skolnick, D. J. Mowbray, I. A. Trojan, S. G. lyapin, L. R. Wilson, M. J. Steer, M. Hopkinson, L. Eaves, and P. C. Main, Phys. Rev. B 60, R2185 (1999).

${ }^{57}$ H. Fu and A. Zunger, Phys. Rev. B 57, R15 064 (1998); L. W. Wang, J. Kim, A. Zunger, ibid. 59, 5678 (1999); L. W. Wang and A. Zunger, ibid. 59, 15806 (1999).

${ }^{58} \mathrm{We}$ can roughly estimate the number of photocreated electronhole pairs in an InP QD, $N_{c}$, under our experimental conditions, using the Fermi golden rule for an optical transition between the discrete energy states of a QD. We consider that the spectral width of the laser pulse is much larger than that of the optical transition, but is less than the spectral distance between the nearest optical transitions. The dipole moment of the optical transition was evaluated from the radiative lifetime determined experimentally for the lowest state of the electron-hole pair, assuming that this time is of the same order of magnitude for the first excited state. Our estimation gives $N_{c}=8$ for the case of the top curve in Fig. 10. Due to saturation of the absorption, the actual number of the carriers must be less. 